\title{
Does pattern matching require the normalization of size and orientation?
}

\author{
MICHAEL KUBOVY \\ Rutgers University, New Brunswick, New Jersey 08903 \\ and \\ PETER PODGORNY \\ Yale University, New Haven, Connecticut 06520
}

\begin{abstract}
Subjects judged whether two visual patterns had the same shape under conditions in which the patterns could vary orthogonally in size and orientation. Analyses of reaction times and errors indicated that, before judging the correspondence between shapes, subjects normalized orientational variation by means of a continuous mental rotation. However, an analogous normalization for variation in size was not detected, and thus no evidence for an internal operation of size scaling was obtained in this experiment. The latter result is at variance with previous findings, and we discuss two possible explanations for this lack of effect.
\end{abstract}

When two patterns differ in ways that could be eliminated by performing a rigid physical transformation, such as rotation or translation, we willingly say that the patterns are the same. In order to reach this decision, perceivers must establish that such physical transformations would in fact bring the patterns into spatial congruence. A recent and influential view is that perceivers perform mental operations that are in many ways analogous to physical transformations and that they decide, by means of these internal operations, whether patterns are identical according to a criterion of congruence (Bundesen \& Larsen, 1975; Bundesen, Larsen, \& Farrell, in press; Cooper \& Shepard, 1978; Dixon \& Just, 1978; Larsen \& Bundesen, 1978; Posner, 1978; Sekuler \& Nash, 1972; Shepard, 1975, 1980). This is the normalization theory.

Normalization is not logically necessary in pattern matching and pattern recognition. In fact, in the early days of cognitive psychology a consensus developed that normalization-as discussed in connection with theories of template matching-was a less desirable solution to the problem of recognition than

This research was supported in part by NIMH Grant MH26573 to Yale University, NIMH Grant MH35137 to Rutgers University, a Rutgers University Research Council grant, and a grant from the Charles and Johanna Busch Memorial Fund. Reprints may be obtained from M. Kubovy, Department of Psychology, Rutgers University (Busch Campus), New Brunswick, New Jersey 08903. P. Podgorny's present address is Department of Psychology, University of Southern California, Los Angeles, California 90007. K. Shahriar ran the experiment; I. L. Kubovy, T. D. Smith, and M. Wexsler ably assisted at various stages. We are grateful to W. R. Garner, M. Sebrechts, A. J. Wearing, and an anonymous reviewer for this journal for helpful comments. feature analysis (Gibson, 1969; Neisser, 1967; Selfridge, 1959). The feature-analytic approach attempted to overcome the problem posed by discrepancies in size and orientation of otherwise identical patterns by describing these patterns in terms of feature lists that were invariant across size and orientation (see, e.g., Selfridge \& Neisser, 1960). Evidence for normalization is therefore important and bears directly on the issue of internal representation. If normalization occurs, it implies that the internal representation being normalized is isomorphic or complementary to its physical referent in some spatial sense (Shepard, 1975, 1980); therefore, any representational formalism based on lists of features or abstract descriptions will be less serviceable theoretically, since representations of this class do not highlight the connectivity and coherence of spatial patterns. Conversely, empirical demonstrations of normalization force us to consider alternative representational systems that are better able to preserve the spatial character of visual information.

We begin this paper by assuming that judgments of identity between two instances of a pattern require that the instances be normalized in a continuous, analog fashion - an assumption that some have questioned (Besner \& Coltheart, 1976; Hinton, 1979)and ask how these two normalizations proceed in real time. That is, when a person sees two visual patterns that differ in both size and orientation, how is this two-dimensional normalization accomplished? A study by Sekuler and Nash (1972) suggests both (1) that our assumption of two-dimensional normalization is reasonable, and (2) that the two normalization operations are independent and add their respective temporal components to measures of real-time 
processing. Using line drawings of rectangles, these authors showed that the time to judge two rectangles as being the same in shape increased linearly with the discrepancy in size between them. In addition, if the two identically shaped rectangles differed in size and orientation (by a 90 -deg rotation), this orientational discrepancy added a constant $70 \mathrm{msec}$ to response time. If these $70 \mathrm{msec}$ can be attributed to the operation of mental rotation, then Sekuler and Nash's results argue for the independence of two mental operations: mental rotation and size scaling (but see Besner, 1978).

After we had concluded the experiment reported in this article, we learned of another, quite similar attempt to study this same question. Bundesen et al. (in press) report data strongly supportive of the concept of two independent and additive normalization operations. The main difference between our study and theirs was that we chose random polygons [which have produced strong effects for rotation (Cooper, 1975) and size (Bundesen \& Larsen, 1975)], and we studied these patterns at five values of orientational discrepancy and five values of size discrepancy, in an orthogonally crossed design; Bundesen et al. used characters (the digits 3,4 , and 7 and the letters $J, P$, and $R$ ), and their mirror inversions, and studied these stimuli at four levels on each of the variables.

\section{METHOD}

\section{Subjects}

Eight subjects participated. Six were undergraduates at Yale University and received credit toward a course requirement as compensation. The remaining two subjects were the authors.

\section{Equipment}

A Gerbrands four-field tachistoscope and digital clock were used for presenting stimuli and for recording reaction times, respectively. Only two of the four fields were needed in this experiment. The subjects pressed one of two telegraph keys to indicate their choice response.

\section{Stimuli}

Two patterns and their mirror images, selected from the set of random polygons studied by Cooper (1975), were used to generate 400 stimuli. Each stimulus consisted of two black patterns on a white background, side by side (see two examples of stimuli in Figure 1).

The cards used in the tachistoscope were $101 \mathrm{~mm}$ in height and $151 \mathrm{~mm}$ in width. If we define the top left of the card to be the origin of a two-dimensional Cartesian coordinate system whose units of distance are millimeters, then the left-hand pattern was always centered at $(38,-50)$ and the right-hand pattern at $(102,-50)$. Across stimuli, the left-hand pattern was a constant size-the smallest size used-which will be designated by the diameter of the circle that would circumscribe the form: $19 \mathrm{~mm}$. The right-hand pattern was, across stimuli, variable in size and ranged from 19 to $95 \mathrm{~mm}$, in steps of $19 \mathrm{~mm}$. Thus, in this experiment, the linear size ratio between the right- and left-hand patterns was $1,2,3,4$, or 5 .

When the left- and right-hand patterns were equal in size $(19 \mathrm{~mm})$, their circumscribing circles were separated by a distance of $44 \mathrm{~mm}$ at their closest points; whereas, when the right-hand pattern assumed its largest value $(95 \mathrm{~mm})$, the two patterns were separated by a distance of $6 \mathrm{~mm}$ (see Figure 1). Viewing distance was approximately $812 \mathrm{~mm}$. At this distance, a $19-\mathrm{mm}$ form
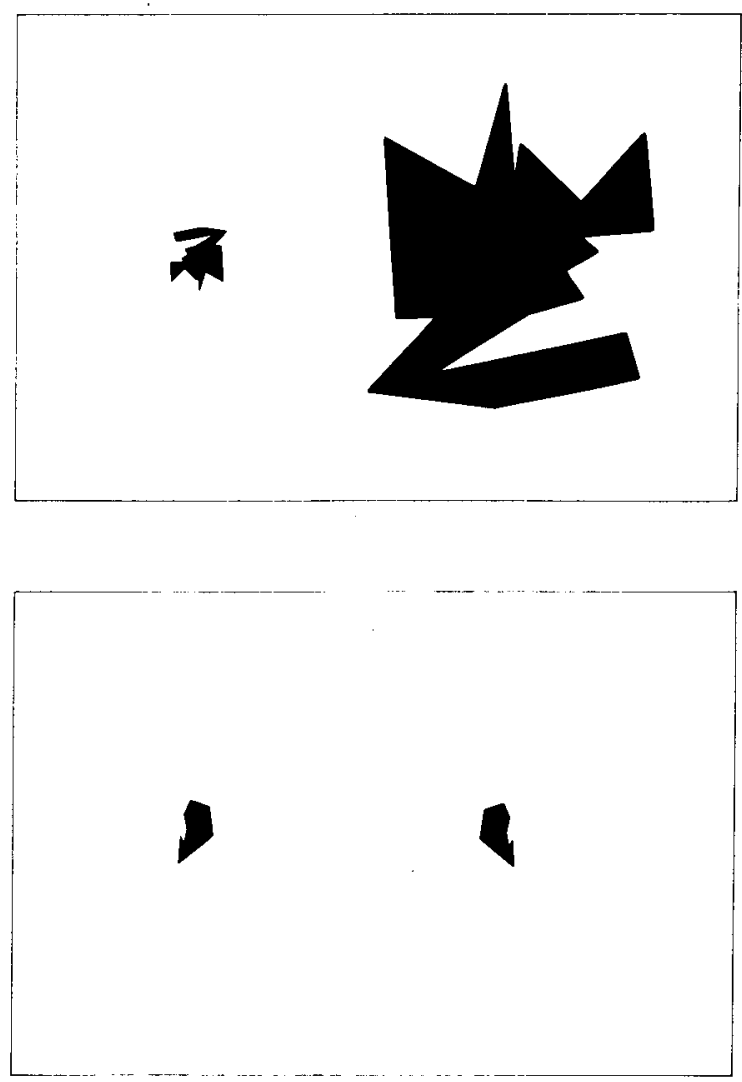

Figure 1. Examples of stimuli used in the experiment. Upper panel: "Same" stimulus; the two patterns are identical except for a size ratio of 1:5 and a rotation of 180 deg. Lower panel: "Different" stimulus; the two patterns differ in that one is the mirror image of the other, but no size or orientational discrepancy has been introduced.

would-if centered along the line of sight-subtend a visual angle of about $1.3 \mathrm{deg}$ and a $95-\mathrm{mm}$ form would subtend an angle of about $6.7 \mathrm{deg}$.

\section{Design}

The experiment was conducted according to a within-subjects complete factorial design. The factors of primary interest were the size and orientation of the two patterns being judged. The lefthand pattern was a constant size but could appear at one of two orientations ( 0 or $180 \mathrm{deg}$ ). Each of the four random polygons was used on $25 \%$ of the stimuli as the left-hand pattern. The righthand pattern could appear at any of five sizes $(19,38,57,76$, or $95 \mathrm{~mm})$ and at any of five orientations $(0,45,90,135$, or $180 \mathrm{deg})$ relative to the left-hand pattern. That is, if the left-hand pattern was presented at $0 \mathrm{deg}$, the right-hand pattern would appear at an orientation between 0 and $180 \mathrm{deg}$ (measured in a clockwise direction); but if the left-hand pattern was presented at $180 \mathrm{deg}$, the right-hand pattern could appear at $180,225,270,315$, or 360 (i.e., 0) deg.

Since factors were crossed, each combination of the five size ratios and the five orientational discrepancies was studied for each pattern at each of the two left-hand orientations. In addition, a stimulus was to be judged "same" if the two patterns were identical, with possible irrelevent variation in size and/or orientation, or the stimulus was to be judged "different" if the two patterns were mirror images, again with possible irrelevant differences in size and/or orientation. Note that the two basic random polygons were never paired to provide a "different" stimulus. Only patterns that were mirror images of each other (after the appropriate size 
scaling and rotation) constituted a "different" stimulus. In all, half of the stimuli were "same" and half "different." Thus, for each subject, the basic design required 5 size ratios by 5 relative orientations by 2 left-hand pattern orientations by 2 left-hand pattern types by 2 versions of left-hand pattern by 2 "same" vs. "different" stimuli $=\mathbf{4 0 0}$ stimuli. Each subject participated in two sessions, consisting of $\mathbf{4 0 0}$ trials each, and lasting $1 \mathrm{~h}$. During each session, a different random permutation of the 400 stimuli was presented. Across the eight subjects, then, 6,400 reaction times were collected.

\section{Procedure}

The subjects sat in front of the tachistoscope and rested each hand on one of the two response keys. The experimenter initiated a trial by saying "now" and pressing a switch that illuminated the first field of the tachistoscope. This field remained illuminated for $1,000 \mathrm{msec}$ and contained two fixation dots, one dot at each location where a pattern would appear. Upon the offset of the first field; a second field-the stimulus field which contained the two patterns to be judged as "same" or "different"-was illuminated. The clock began timing from the onset of the stimulus field. This stimulus field remained illuminated for $2,000 \mathrm{msec}$, and the subject's choice response stopped the clock. All subjects used their right hands to press the switch that indicated the patterns were the "same" and their left hands to press the switch that indicated the patterns were "different."

The subjects were told that their task was to judge the two patterns as being "same" or "different" as quickly and accurately as possible and that to be considered "same," the patterns must be superimposable in the picture plane after (possibly) a corrective adjustment in the size and/or orientation of one pattern. That is, the subjects were informed that differences in size and orientation were irrelevant to the judgment of sameness. In addition, the subjects were told that stimuli requiring a "same" response would occur as often, on the average, as stimuli requiring a "different" response.

At the beginning of the experiment, each subject was given 20 practice stimuli that were selected randomly from the total set of experimental stimuli. Experimental trials on which errors occurred were repeated later in the session so that a correct reaction time was available for every cell in the design.

\section{RESULTS}

Mean reaction time for correct responses provided the primary dependent measure for analysis, and we will concentrate our analysis on the "same" stimuli, since the concept of normalization is most readily defined when two patterns can be brought into congruence and thereby receive a judgment of "same."

\section{Preliminary Analyses}

The mean error rate was $4.1 \%$, with individual subjects ranging from $.9 \%$ to $8.0 \%$. The error rates were somewhat higher for "same" stimuli (i.e., stimuli on which subjects incorrectly responded "different") than for "different" stimuli (4.9\% vs. $3.2 \%$, respectively). The error rates were highly correlated with the corresponding reaction times. The productmoment correlation between errors to "same" stimuli and correct "same" reaction times over the 25 conditions of the experiment ( 5 sizes by 5 orientations) was .89; the analogous correlation between errors to "different" stimuli and correct "different" reaction times was .65 .
The mean reaction time for the "different" responses was longer than the mean for the "same" response $(1,873 \mathrm{msec}$ vs. $1,518 \mathrm{msec})$; but the times for the two kinds of response were highly correlated over the 25 conditions and produced a product-moment correlation of .97 .

We conclude from these analyses that the reaction time data for "different" responses as well as the error data present essentially the same picture as the reaction time data for "same" responses.

\section{Analysis of "Same" Reaction Times}

Reaction time data for "same" responses are presented in two different ways in Figure 2. The left panel of Figure 2 shows a group of five functions relating reaction time and angular departure. Each of the five functions represents a different value of size discrepancy. A three-factor (Size by Angle by Session) repeated-measures ANOVA established the effect of angle $[F(4,28)=24.83, M S e=468,914, p<.001]$, and two linear contrasts showed that there was a highly significant linear trend as a function of angle $[F(1,28)=97.37, \mathrm{MSe}=468,914, \mathrm{p}<.001]$, but detected no significant quadratic trend $[F(1,28)=1.73$, $\mathrm{MSe}=468,914]$. The fact that the five functions in the left panel fall very close to one another and frequently crisscross indicates that there is no consistent effect of size disparity on reaction time. This is even more readily seen if we replot the data, as in the right panel of Figure 2, so that the size variable is measured along the abscissa. The flatness of the five functions in the right panel of Figure 2 shows, again, that size is not influencing reaction time. This conclusion is reinforced statistically by the lack of a main effect of size in the omnibus ANOVA $[F(4,28)=.93$, $\mathrm{MSe}=27,346$ ] and by the lack of any linear trend of reaction time as a function of size $[F(1,28)=.25$, $\mathrm{MSe}=27,346]$. Furthermore, the omnibus ANOVA showed no interaction of size and angle $[F(16,112)=$ $1.14, \mathrm{MSe}=23,404]$.

\section{The Power of Our Test to Detect an Effect of Size}

Since other experimenters have found that size disparity delays identity judgments, we turn now to the issue of the power of our analysis. In calculating power, we made the following assumptions: First, we used as an estimate of the range of difference we could expect to find in reaction time, for the range of sizes we studied, Bundesen and Larsen's (1975) range of reaction times across size ratios 1 to 5 (viz., a range of approximately $100 \mathrm{msec}$ in their Experiment 2, an experiment quite comparable in methodology to ours). Second, we assumed that we were assessing the power of our test to detect differences among means equally spaced over a $100-\mathrm{msec}$ range irrespective of order (an extremely conservative assumption, since in actuality we are interested in a linear trend). Third, we adopted an alpha level of .05 . 

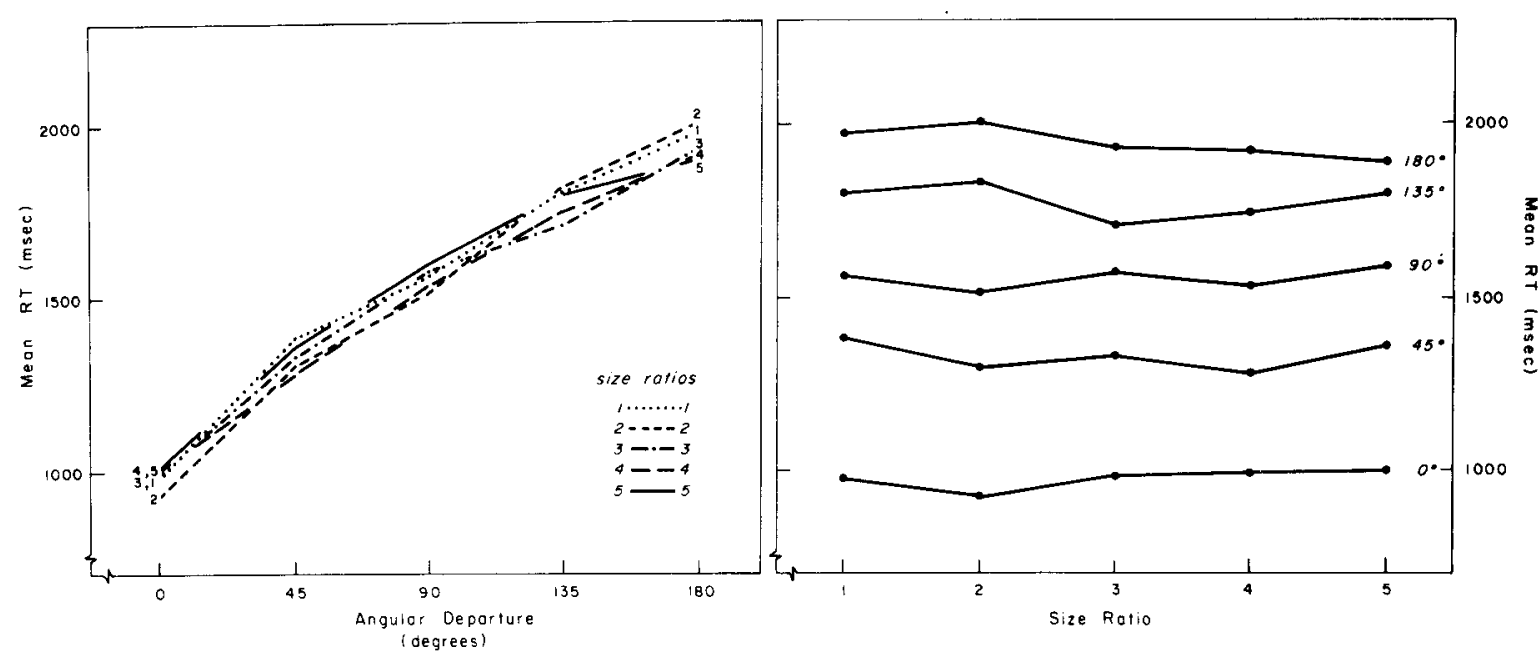

Figure 2. Two representations of the reaction times for "same" responses to "same" stimuli. Left panel: Mean reaction time as a function of angular departure. Size ratio is the parameter. Right panel: Mean reaction time as a function of size ratio. Angular departure is the parameter.

We calculated power according to a procedure recommended by Cohen (1969, chap. 8). Using the meansquare error obtained in our ANOVA for the Size by Subject interaction $(\mathrm{MSe}=\mathbf{2 7 , 3 4 6}$ ) in order to obtain a value of $d$, the standardized range of population means $\left[d=100 /\left(27,346^{1 / 2}\right)=.605\right]$, we then obtained a value of $f$, the effect-size index, under the assumptions that the means for the five size ratios would be equally spaced within the 100 -msec range, namely $f=.214$. Finally, we used Laubscher's square-root normal approximation of the noncentral F (Cohen, 1969, p. 405) and obtained a unit-normal percentile value of 1.299 , which corresponds to a power of .90 .

Thus, we conclude that under the circumstances of our test, and setting alpha at .05 , the probability of detecting a distribution of means such as reported by Bundesen and Larsen (1975) would have been considerably better than .90 . Therefore, we are confident that we are not erroneously accepting the null hypothesis of no effect of size. Our confidence is increased by the observation that the "different" response times and the error data converge on the same conclusion.

\section{DISCUSSION}

The left panel of Figure 2 makes very clear that, for "same" responses, reaction time increases linearly with the angular departure between the two patterns. The linearity of this result suggests that (1) subjects are normalizing the two patterns by mentally rotating one pattern into the orientation of the other and (2) the average rate of this mental rotation is approximately constant over the range of orientational discrepancies studied here. The right panel of Figure 2 makes clear that, for "same" responses, reaction time is unaffected by the degree of disparity in size between the two patterns. Thus, there is no evidence in these data for a mental operation of size scaling that normalizes the size of one pattern in order to match the size of the other before the patterns are judged for congruence.

We had expected to find an effect of size, just as did Besner and Coltheart (1976), Bundesen and Larsen (1975), Bundesen et al. (in press), and Sekuler and Nash (1972). We are uncertain why we did not. There are several possibilities.

First, our reaction times range from 900 to 2,000 msec, whereas Besner and Coltheart's do not exceed $600 \mathrm{msec}$, Bundesen and Larsen's are less than 1,100 msec, Sekuler and Nash's are all below $500 \mathrm{msec}$, and Bundesen et al.'s are not greater than $900 \mathrm{msec}$. Apparently, the time-consuming operation of mental rotation is inflating our subjects' reaction times; and perhaps it is the occurrence of these long times that obscures in some way the effect of size. Although our previous analyses were quite powerful in their ability to detect an overall size effect, these analyses did not search for a plausible, but subtle, trend that might be present in these data. Supposecontrary to Bundesen et al. (in press) and Sekuler and Nash (1972) - that the effects of size and orientation are not additive but are interactive in the following way and for the following reason. If normalizations for size and orientation were to occur in parallel and if the latter were much more timeconsuming than the former, then subjects would finish the size scaling before they finished the mental rotation, even though they could not respond until they had finished both. By this view, whenever size and orientation both vary, the latter variable will dominate the former. Therefore, the data most relevant to the possible existence of a size effect are those trials on which no rotation was required. These data are shown as the bottom function in the right panel of Figure 2. But, as can be seen in Figure 2, even these nonrotated stimuli produce scant evidence for 
an effect of size. We formed an orthogonal contrast to test for the possibility that the bottom (no-rotation) function had a different linear slope from the average slope of the four functions above it in the right panel of Figure 2, but this contrast was not significant $[F(1,112)=1.89, M S e=23,404]$. Thus, we are left with the same conclusion: There is no solid evidence for size scaling in these data.

Second, there exists evidence that not all tasks that could in principle be done via normalization are actually done in this fashion. For example, Cooper and Shepard (1973) have argued that mental rotation cannot be the operative mechanism behind the subjects' identification of physically rotated letters because identification times are much faster than the times associated with the mental rotation of letters. Likewise, Corballis, Zbrodoff, Shetzer, and Butler (1978) have presented empirical evidence which suggests that the identity of letters and digits is recognized before their orientation is perceived and before they are mentally rotated (if they are). It would seem that mental rotation is used in tasks in which the subject cannot make the required judgment by any simpler categorical rule. So, for example, one need not rotate the tilted image of a man in order to decide that it is not the same as the upright image of a woman; an analysis of the distinctive physical features of each is sufficient to answer the question of identity and is possibly less demanding than mental rotation.

In this vein, one explanation for the lack of a size effect in our experiment is that the conditions we used were not adequate to induce subjects to adopt a size-scaling strategy. The only design characteristics of our experiment that might distinguish it from other experiments that have shown an effect of size are (1) the fact that we used only two different random polygons (and their mirror inversions), and (2) the fact that the size of the left-hand pattern in a stimulus was always a constant size. Convincing explanations of why either of these design characteristics should influence the subjects' use of size scaling or of why they should influence only size scaling and not orientational normalization require more information than we currently possess. Consistent, however, with our belief that different conditions might be necessary to induce mental rotation and size scaling is the observation that size and orientation play different roles in the perception of visual form. Size is easily separable from form, whereas orientation can interact with form, even to the extent of changing a pattern's name when it is rotated (Goldmeier, 1972, chap. 6; Rock, 1973).

In summary, our experiment suggests that size normalization is not always necessary for pattern matching. An alternative proposal-namely, that size normalization can be performed in parallel with other, more time-consuming mental operations such as rotation-is not supported by our data or those of Bundesen et al. (in press) or Sekuler and Nash (1972).
It is most likely that subjects are induced to adopt a mental-rotation strategy or a size-scaling strategy by certain stimulus or task variables. Furthermore, these inducing variables may not be the same for the two internal processes.

\section{REFERENCES}

Besner, D. Pattern recognition: Are size and orientation additive factors? Perception \& Psychophysics, 1978, 23, 93.

Besner, D., \& Coltheart, M. Mental size scaling examined. Memory \& Cognition, 1976, 4, 525-531.

Bundesen, C., \& Larsen, A. Visual transformation of size. Journal of Experimental Psychology: Human Perception and Performance, 1975, 1, 214-220.

Bundesen, C., Larsen, A., \& Farrell, J. E. Visual transformations of size and spatial orientation: Spatial imagery and perceptual processes. In A. Baddeley \& J. Long (Eds.), Attention \& performance IX. Hillsdale, N.J: Erlbaum, in press.

COHEN, J. Statistical power analysis for the behavioral sciences. New York: Academic Press, 1969.

Cooper, L. A. Mental transformation of random two-dimensional shapes. Cognitive Psychology, 1975, 7, 20-43.

Cooper, L. A., \& Shepard, R. N. Chronometric studies of the rotation of mental images. In W. G. Chase (Ed.), Visual information processing. New York: Academic Press, 1973.

Cooper, L. A., \& Shepard, R. N. Transformations on representations of objects in space. In E. C. Carterette \& M. Friedman (Eds.), Handbook of perception (Vol. 8) Space and object perception. New York: Academic Press, 1978.

Corballis, M. C., Zbrodoff, N. J., Shetzer, L. I., \& Butler, P. B. Decisions about identity and orientation of rotated letters and digits. Memory \& Cognition, 1978, 6, 98-107.

Dixon, P., \& Just, M. A. Normalization of irrelevant dimensions in stimulus comparisons. Journal of Experimental Psychology: Human Perception and Performance, 1978, 4, 36-46.

GiBson, E. J. Principles of perceptual learning and development. New York: Appleton-Century-Crofts, 1969.

Goldmeier, E. Similarity in visually perceived forms. Psychological Issues, 1972, 8(Whole No. 29). (Originally published in 1936)

Hinton, G. Some demonstrations of the effects of structural descriptions in mental imagery. Cognitive Science, 1979, 3, 231-250.

LARSEN, A., \& Bundesen, C. Size scaling in visual pattern recognition. Journal of Experimental Psychology: Human Perception and Performance, 1978, 4, 1-20.

Neisser, U. Cognitive psychology. Englewood Cliffs, N.J: Prentice-Hall. 1967.

Posnen, M. I. Chronometric explorations of mind. Hillsdale, N.J: Erlbaum, 1978.

Rock, I. Orientation and form. New York: Academic Press, 1973.

Sexuler, R., \& NAsh, D. Speed of size scaling in human vision. Psychonomic Science, 1972, 27, 93-94.

Selfridge, O. G. Pandemonium: A paradigm for learning. In The mechanism of thought processes (Proceedings of a symposium, National Physical Laboratory, Teddington, England). London: Her Majesty's Stationery Office, 1959.

Selfridge, O. G., \& Neisser, U. Pattern recognition by machine. Scientific American, 1960, $203(2), 60-68$.

SHEPARD, R. N. Form, formation, and transformation of internal representations. In $\mathbf{R}$. Solso (Ed.), Information processing and cognition: The Loyola symposium. Hillsdale, N.J: Erlbaum, 1975.

ShePARD, R. N. Psychophysical complementarity. In M. Kubovy \& J. R. Pomerantz (Eds.), Perceptual organization. Hillsdale, N.J: Erlbaum, 1981.

(Manuscript received July 28, 1980; revision accepted for publication March 26, 1981.) 